\title{
Quercetin Alleviates Osteoarthritis Progression in Rats by Suppressing Inflammation and Apoptosis via Inhibition of IRAKI/NLRP3 Signaling
}

\author{
Wenjun $\mathrm{Li}^{1}$ \\ Yeyang Wang' \\ Yaqin Tang ${ }^{2}$ \\ Hanyu Lu' \\ Yong $\mathrm{Qi}^{\mathrm{I}}$ \\ Guitao Li ${ }^{1}$ \\ Hebei $\mathrm{He}^{\prime}$ \\ Fanglian Lu' \\ Yixin Yang \\ Hongtao Sun (iD) \\ 'Department of Orthopedics, \\ Guangdong Second Provincial General \\ Hospital, Guangzhou, 510317, \\ Guangdong Province, People's Republic \\ of China; ${ }^{2}$ Department of Internal \\ Medicine, The First Affiliated Hospital of \\ Guangzhou University of Traditional \\ Chinese Medicine and Pharmacology, \\ Guangzhou, Guangdong Province, \\ 5 10405, People's Republic of China
}

Correspondence: Hongtao Sun

Department of Orthopedics, 12th Floor, Building 3, Guangdong Second Provincial

General Hospital, 466 Xingang Zhong

Road, Haizhu District, Guangzhou,

5I0317, Guangdong Province, People's

Republic of China

Tel +86 I38088499/8

Email shtgd2h@126.com

Hanyu Lu

Department of Orthopedics, 12th Floor, Building 3, Guangdong Second Provincial General Hospital, 466 Xingang Zhong

Road, Haizhu District, Guangzhou,

Guangdong Province, 510317, People's

Republic of China

Tel +86 I8925077|62

Email luhanyu0129@I63.com
Introduction: Quercetin was recently reported to help protect against osteoarthritis (OA) progression, but the molecular mechanism for that protective affect remains unclear.

Methods: Here, OA model rats were intraperitoneally injected with quercetin, and the severity of cartilage damage in the rats was evaluated by H\&E, Safranin O, and Toluidine blue, as well as by using the Osteoarthritis Research Society International (OARSI) Scoring System. Additionally, rat chondrocytes were treated with quercetin and then stimulated with IL-1 $\beta$. The levels of pro-inflammatory cytokines (IL-1 $\beta$, IL-18, and TNF- $\alpha$ ) were detected by ELISA.Cell apoptosis was evaluated by flow cytometry and Hoechst staining. ROS levels were measured using a DCFH-DA probe. Protein expression was evaluated by Western blotting, immunohistochemical staining, and immunofluorescence.

Results: Our data showed that quercetin attenuated the degeneration and erosion of articular cartilage, suppressed inflammation and apoptosis, and downregulated the levels of IRAK1, NLRP3, and caspase-3 expression. In vitro data showed that overexpression of NLRP3 could reverse the suppressive effect of quercetin on IL-1 $\beta$-induced rat chondrocyte injuries. Importantly, rescue experiments confirmed that quercetin inhibited IL- $1 \beta$-induced rat chondrocyte injuries in vitro by suppressing the IRAK1/NLRP3 signaling pathway.

Conclusion: Our study indicated that quercetin inhibits IL-1 $\beta$-induced inflammation and cartilage degradation by suppressing the IRAK1/NLRP3 signaling pathway.

Keywords: quercetin, osteoarthritis, IRAK1, NLRP3, inflammation, apoptosis

\section{Introduction}

Osteoarthritis (OA) is a joint disorder that commonly presents in middle-aged and elderly individuals, and is primarily characterized by impaired joint function, osteophyte formation, and articular cartilage degradation. ${ }^{1,2}$ Although surgical treatments (arthroplasty, joint osteotomy, and arthroscopic surgery) and nonsurgical therapies (oral administration of non-steroidal anti-inflammatory drugs or paracetamol and intra-articular injection of corticosteroids) for OA can attenuate clinical symptoms to some degree, those treatments are unsatisfactory for improving injured joint tissues or slowing OA progression. ${ }^{3-5}$ Therefore, there remains an urgent need to understand the molecular mechanisms of OA in order to develop new strategies for its treatment.

Chondrocytes, as the main cells of articular cartilage, play a crucial role in maintaining a balance between extracellular matrix and tissue homeostasis. ${ }^{6}$ Thus, abnormal chondrocyte function and impaired chondrocyte survival can cause the 
degeneration of the articular cartilage, which might be a major factor involved in the occurrence and development of OA. Interleukin-1 receptor-associated kinase-1 (IRAK1) is a serine-threonine kinase that mediates tolllike receptor (TLR) and interleukin-1 (IL-1) signaling pathways, which have been reported to regulate innate immunity and inflammation. Inhibition of IRAK1 has possible therapeutic benefits when treating tumors, ${ }^{7,8}$ cardiovascular, and inflammatory diseases. ${ }^{9}$ The nucleotide binding and oligomerization, leucine-rich repeat, pyrin domain-containing 3 (NLRP3) inflammasome is composed of NLRP3, which is an apoptosis-associated speck-like protein containing a caspase recruitment domain (ASC) and procaspase-1. ${ }^{10}$ A previous study demonstrated that IRAK1 is crucial for rapid activation of NLRP3 via the MyD88 pathway. $^{11}$ Lin et al $^{10}$ showed that primerdependent activation of the NLRP3 inflammasome occurs independent of IRAK-1. Moreover, Kang et $\mathrm{al}^{12}$ showed that blockage of TLR4/6-IRAK4/1 signaling to suppress NLRP3 inflammasome activation might protect against fructose-induced cardiac inflammation and fibrosis. Interestingly, inhibition of IRAK1 phosphorylation was found to be involved in the mechanism by which delphinidin inhibits IL- $1 \beta$-induced expression of COX-2 and PGE2 production in human chondrocytes. ${ }^{13}$ Furthermore, NLRP3 inflammasomes have been suggested as novel biomarkers for use in managing $\mathrm{OA},{ }^{14}$ because they play a role in the protective effects of icariin ${ }^{15}$ and curcumin ${ }^{16}$ against OA. Nevertheless, it remains unclear how IRAK1 regulates NLRP3 inflammasomes during OA progression.

Quercetin (3,3', 4',5,7-pentahydroxyflavone), a naturally occurring flavonoid found in many types of fruits and vegetables, is known for its various beneficial characteristics, and especially its anti-inflammatory and anti-oxidant properties when used to treat degenerative diseases. ${ }^{17,18}$ In recent years, quercetin has been widely reported to protect against the inflammation, apoptosis, and oxidative stress associated with OA. Qiu et al ${ }^{19}$ confirmed that the therapeutic effects of quercetin on $\mathrm{OA}$ might result from its effects on the adenosine monophosphate-activated protein kinase/sirtuin 1 (AMPK/SIRT1) signaling pathway. Feng et $\mathrm{al}^{20}$ showed that quercetin could attenuate oxidative stress-induced apoptosis via SIRT1/AMPI-mediated inhibition of ER stress, and prevent OA progression in a rat model. Consistent with those findings, $\mathrm{Hu}$ et $\mathrm{al}^{21}$ reported that quercetin exerted chondroprotective effects by modulating the polarization of synovial macrophage to M2 macrophages. However, to the best our knowledge, it still not unclear whether the protective effect of quercetin on chondrocytes is related to IRAK1/NLRP3.

Based on these findings, we constructed an OA rat model, isolated rat chondrocytes, and constructed an in vitro $\mathrm{OA}$ model by incubating rat chondrocytes with IL-1 $\beta$. We then used these models to explore the effects of quercetin on inflammation, apoptosis, and ROS production in vivo and in vitro. Furthermore, we investigated whether IRAK1/ NLRP3 was involved in the downstream mechanism underlying the therapeutic effects of quercetin on OA pathogenesis.

\section{Materials and Methods} OA Rat Model and Drug Administration

A total of 40 male Sprague-Dawley (SD) rats (5 weeks old; weight range, 240-270 g) were purchased from SinoBritish Sippr/BK Lab Animal Ltd. (Shanghai, China). Quercetin was dissolved in DMSO. The rats were housed in a room with controlled temperature and a 12-h light/ dark cycle; food and water were available ad libitum. OA was induced by surgical transection of the right anterior cruciate ligament (ACL) as described in a previous study. $^{22}$ Starting at two weeks after ACL surgery, rats in the experimental group received an intraperitoneal injection of low-dose (LD) quercetin (Volume: $500 \mu \mathrm{L}$, dose: $50 \mathrm{mg} / \mathrm{kg}$, concentration: $49.5 \mathrm{nM}$, Sigma-Aldrich, St Louis, MO, USA) or high-dose (HG) quercetin (Volume: $500 \mu \mathrm{L}$, dose: $100 \mathrm{mg} / \mathrm{kg}$, concentration: $99 \mathrm{nM}$ ) once daily for 12 consecutive weeks. The three experimental groups included OA, OA + LD-quercetin, and OA + HDquercetin groups, with ten rats in each group. Meanwhile, rats in a sham control group received arthrotomy without transection of the anterior cruciate ligament in the right knee joint $(n=10)$. At 12 weeks, all rats were sacrificed by an overdose pentobarbital, and samples of articular cartilage were harvested for further analysis. The protocols for all animal experiments were reviewed and approved by the Ethics Committee of Guangdong Second Provincial General Hospital (2019-KZ-10-28). This animal experiments was performed according to the guideline of Animal Care and Use Committee of Guangdong Second Provincial General Hospital.

\section{Histological Analysis}

Samples of articular cartilage from each group were fixed in $10 \%$ paraformaldehyde for $24 \mathrm{~h}$ and then decalcified in $20 \%$ EDTA for 1 month. Next, the cartilage tissues were 
dehydrated in a graded ethanol series, embedded in paraffin, and cut into $5.0 \mu \mathrm{m}$ sections as previously described. ${ }^{23}$ Subsequently, the degeneration of articular cartilage was evaluated by five types of staining techniques. Briefly, for hematoxylin and eosin (H\&E) staining, the cartilage samples were stained with Harris alum hematoxylin for $5 \mathrm{~min}$ and then stained with eosin for $40 \mathrm{~s}$, followed by observation of the chondrocyte nucleus under a microscope. For Safranin O staining, the samples were stained with $0.02 \%$ fast green for 30 minutes, $1 \%$ acetic acid for 10 seconds, and $1.5 \%$ Safranin $\mathrm{O}$ for 3 minutes. After being dehydrated and mounted with neutral balsam, the cartilage samples were observed under a microscope. The general histological features of cartilage and bone samples were assessed by toluidine blue staining $(0.04 \%$ toluidine blue in $0.2 \mathrm{M}$ acetate buffer, $\mathrm{pH} 3.75-4.25,5 \mathrm{~min})$. Two independent experienced researchers who were blinded to the study analyzed cartilage degenerative changes based on the Osteoarthritis Research Society International (OARSI) Scoring System as previously described. ${ }^{24}$

\section{Immunohistochemical Staining}

In brief, the paraffin-embedded cartilage tissues were cut into $20 \mu \mathrm{m}$ sections. After xylene dewaxing and dehydration in a graded alcohol series, the sections underwent antigen retrieval in sodium citrate buffer for $48 \mathrm{~h}$ at room temperature. Next, the slices were blocked with $5 \%$ BSA in TBST for $30 \mathrm{~min}$ and subsequently incubated with an NLRP3 primary antibody (1:100 dilution; Abcam, Cambridge, UK) and Caspase 3 (1:200, Boster, Hubei, China) at $4^{\circ} \mathrm{C}$ overnight; after which, they were incubated with a horseradish peroxidase-conjugated secondary antibody (1:5000 dilution; Abcam) at room temperature for 2 h. The slices were then visualized with 3,3'diaminobenzidine chromagen $(1 \mathrm{mg} / \mathrm{mL})$ at room temperature for $5 \mathrm{~min}$. The stained images were photographed using a light microscope and analyzed by Image-Pro Plus Software version 6.0 (Media Cybernetics, Rockville, MD, USA).

\section{Isolation and Culture of Chondrocytes}

Primary chondrocytes were isolated and cultured as previously reported. ${ }^{25}$ In brief, cartilage tissues from the rat hip joint were cut into small pieces under sterile conditions. After being washed 5 times with PBS, the cartilage pieces were digested in $0.25 \%$ trypsin-EDTA solution for $1 \mathrm{~h}$ and then incubated with Dulbecco's Modified Eagle's Medium/Nutrient Mixture F-12 (DMEM/F-12) containing
$0.2 \%$ collagenase type II for $4 \mathrm{~h}$. Following centrifugation at $1200 \mathrm{rpm}$ for $5 \mathrm{~min}$, the released chondrocytes were seeded into $25 \mathrm{~cm}^{2}$ cell flasks and cultured in DMEM-F12 supplemented with 10\% FBS and 1\% penicillin plus streptomycin. Chondrocytes at passage 2 with $80-90 \%$ confluence were used for in vitro experiments.

\section{Cell Treatment and Transfection}

The isolated chondrocytes were seeded into culture plates containing DMEM-F12 complete medium and grown to $80 \%$ confluence. The chondrocytes were then serumstarved overnight and treated with $8 \mu \mathrm{M}$ quercetin for 2 $\mathrm{h}$; after which, they were stimulated with IL-1 $\beta$ (10 ng/ $\mathrm{mL}$, Pepro Tech, NJ, USA) at $37^{\circ} \mathrm{C}$ in a $5 \% \mathrm{CO}_{2}$ atmosphere. For cell transfection, chondrocytes in the IL-1 $\beta+$ quercetin group were transiently transfected with an NLRP3 or IRAK1 overexpression plasmid or empty vector, or co-transfected with siRNA targeting NLRP3 (siNLRP3) and an IRAK1 overexpression plasmid by using Lipofectamine 2000 (Invitrogen, Carlsbad, CA, USA), according to the manufacturer's instructions. The chondrocytes were harvested after $48 \mathrm{~h}$ of transfection.

\section{Enzyme-linked Immunosorbent Assay (ELISA)}

Pro-inflammatory cytokines (IL-1 $\beta$, IL-18, and TNF- $\alpha$ ) were detected by using conventional ELISA kits (R\&D systems, Minneapolis, MN, USA) according to instructions provided by the manufacturer.

\section{Cell Viability Assay}

Cell viability was measured using a cell counting kit-8 (CCK-8, Boster, Wuhan, China) according to the standard protocol. Briefly, chondrocytes from different groups were seeded into the wells of 96-well plates at a density of $1 \times 10^{4}$ cells per well and then incubated with $10 \mu \mathrm{L}$ of CCK-8 solution for 24, 48, 72, and $96 \mathrm{~h}$, respectively. At each time point, the absorbance at a wavelength of $450 \mathrm{~nm}$ (OD 450nm) was determined with a microplate reader.

\section{Apoptosis Analysis}

The apoptosis of rat chondrocytes was assessed by flow cytometry analysis performed with an Annexin V-FITC/ propidium iodide (PI) apoptosis kit. Briefly, chondrocytes from the different groups were harvested and re-suspended in $500 \mu \mathrm{L}$ of binding buffer. After being stained with $5 \mu \mathrm{L}$ of Annexin $\mathrm{V}$ and $10 \mu \mathrm{L}$ of PI for $20 \mathrm{~min}$ at room 
temperature, the apoptotic cells were detected with a $\mathrm{BD}$ FACSCalibur flow cytometer (BD Biosciences, Franklin Lakes, NJ, USA).

\section{Hoechst Staining}

Chondrocytes from the different groups were seeded into the wells of 96-well plates at a density of $7 \times 10^{3}$ cells per well, and cultured overnight at $37^{\circ} \mathrm{C}$. After removing the culture medium, the chondrocytes were fixed with $4 \%$ paraformaldehyde at $4^{\circ} \mathrm{C}$ for $1 \mathrm{~h}$, permeabilized with saponin, and then stained with Hoechst 33342 (Beyotime Institute of Biotechnology) for $30 \mathrm{~min}$ at room temperature in the dark. The stained cells were visualized using a fluorescence microscope (Nikon Corporation) at $\times 200$ magnification.

\section{Measurements of Intracellular ROS}

To determine the intracellular levels of ROS, chondrocytes from different groups were washed with PBS and then incubated with DCFH-DA (20 $\mu \mathrm{M}$, final concentration) for $15 \mathrm{~min}$ in the dark. Next, the cells were washed with PBS and suspended by trypsinization. Following centrifugation $\left(4^{\circ} \mathrm{C}, 3000 \mathrm{rpm}, 5 \mathrm{~min}\right)$, the cells were resuspended in $1 \mathrm{~mL}$ of PBS and analyzed by flow cytometry.

\section{Western Blot Analysis}

The total proteins were extracted with RIPA lysis buffer containing a protease inhibitor and phosphatase inhibitor cocktail (Roche, Stockholm, Sweden), and the protein concentration in each extract was determined by using a BCA protein assay kit (Beyotime Institute of Biotechnology). Next, an equal amount of protein from each extract was separated by $12 \%$ SDS-polyacrylamide gel electrophoresis. The separated protein bands were transferred onto polyvinylidene fluoride (PVDF) membranes (Bio-Rad, Hercules, CA, USA), which were subsequently blocked with $5 \%$ non-fat milk in Tris-buffered saline tween (TBST) for $2 \mathrm{~h}$. The membranes were then incubated overnight with primary antibodies against IRAK1, NLRP3, ASC, iNOS, COX-2 and caspase-3, and GAPDH at $4^{\circ} \mathrm{C}$. After being washed with TBST, the membranes were incubated with a horseradish peroxidase-conjugated secondary antibody for $2 \mathrm{~h}$ at room temperature, and the immunostained protein bands were visualized by enhanced chemiluminescence (ECL, Pierce, Waltham, MA, USA).

\section{Immunofluorescence}

Immunofluorescence staining of NLRP3 was performed to analyze the levels of NLRP3 protein expression. In brief, chondrocytes from the different groups were fixed with $1 \mathrm{~mL}$ of $4 \%$ PFA for $25 \mathrm{~min}$, permeated with $0.2 \%$ Triton $\mathrm{X}-100$ for $5 \mathrm{~min}$, and blocked with goat serum for 1 h. Next, the cells were incubated overnight with primary antibodies against NLRP3, followed by incubation with Alexa Fluor 555-conjugated secondary antibodies. The cellular nuclei were stained with DAPI. Images of the stained cells were obtained with a fluorescence microscope (Olympus).

\section{Statistical Analysis}

All statistical analyses were performed using GraphPad Prism 6.0 software (GraphPad Software, Inc.). All quantitative data are expressed as a mean value \pm standard deviation of data obtained from three independent experiments. Differences among groups were analyzed by oneway ANOVA, followed by Tukey's post hoc test. A P-value $<0.05$ was considered to be statistically significant.

\section{Results}

\section{Quercetin Suppressed OA Progression and Inflammation in vivo}

We first investigated the effects of quercetin on OA progression by establishing a rat $\mathrm{OA}$ model and giving the rats an intraperitoneal injection of quercetin for 4 consecutive weeks. After harvesting the knee joints, histopathological were performed by using H\&E staining, Toluidine blue staining and Safranin O staining. H\&E staining showed the normal morphology of joints in the sham group, whereas knee joints in the OA model rats exhibited cartilage defects and narrowed joint spaces (Figure 1A). Notably, quercetin treatment attenuated the degeneration and erosion of articular cartilage, and that attenuation effect was more obvious in the high-dose group than the low-dose group (Figure 1B and C). The OARSI scores in the OA group were significantly higher than those in the sham group, while rats in the quercetin treatment groups had much lower OARSI scores than rats in the OA group, in a dose-dependent manner (Figure 1D). Moreover, we found that the increase in pro-inflammatory cytokine (IL$1 \beta$, IL-18, and TNF- $\alpha$ ) release in the OA group compared with the sham group was significantly reduced after treatment with either low dose or high dose quercetin 


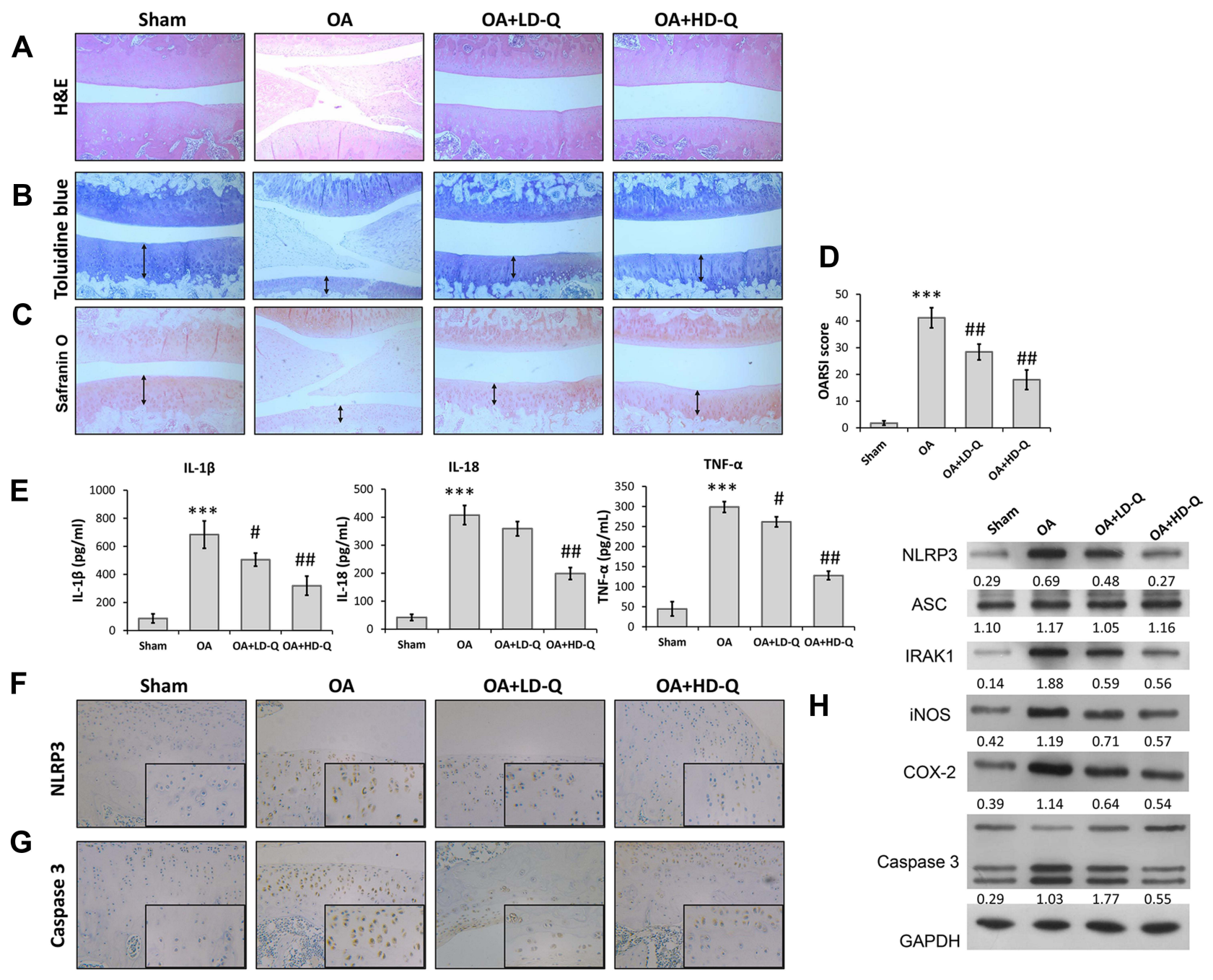

Figure I Quercetin alleviated OA progression and suppressed inflammation in vivo. Rats were assigned to 4 separate groups: a sham, OA, OA + LD-Q, and OA + HD-Q group. (A-C) The degree of cartilage destruction and joint spacing in each group was evaluated at 12 weeks by H\&E staining (A), toluidine blue (B) and safranin $O$ staining (C). Arrow in (B and C) showed the distribution of chondrocyte. (D) Osteoarthritis Research Society International (OARIS) scores for articular cartilage in the 4 groups. (E) The levels of pro-inflammatory cytokines (IL-I $\beta$, IL-I8, and TNF- $\alpha$ ) were measured by ELISA. (F and $\mathbf{G})$ Immunohistochemical staining for NLRP3 expression (F) and Caspase 3 (G). (H) The levels of NLRP3, ASC, IRAKI, iNOS, COX-2 and caspase 3 proteins were measured by Western blotting. Data represent mean \pm standard deviation. *** $\mathrm{p}<0.001$, compared with sham; ${ }^{\#}<0.05,{ }^{\# \#} \mathrm{p}<0.01$, compared with OA.

(Figure 1E). Immunohistochemical staining showed that quercetin significantly decreased the numbers of NLRP3 (Figure 1F) and Caspase 3 (Figure 1G) positive cells in rat articular cartilage when compared with the OA group. We also examined the levels of IRAK1, NLRP3, ASC, iNOS, COX-2 and pro-apoptotic caspase-3 protein expression by Western blotting (Figure 1H). Our results showed that treatment with the high dose of quercetin suppressed IRAK1, NLRP3, and caspase-3 expression, but did not obviously affect ASC expression in the OA group. These data suggested that quercetin could enhance the structural integrity of rat joint cartilage and alleviate $\mathrm{OA}$ progression.

\section{Quercetin Attenuated IL-I $\beta$-Induced Inflammation and Apoptosis in Rat Chondrocytes}

To ascertain the protective role of quercetin in OA pathogenesis, we isolated rat chondrocytes and used them to mimic OA chondrocytes by incubation with IL-1 $\beta$ (10 ng/ $\mathrm{mL}$ ) for $24 \mathrm{~h}$. $\mathrm{IC}_{50}$ of OA chondrocytes to quercetin was detected and result showed that the concentration of $\mathrm{IC}_{50}$ of OA chondrocytes was $11.58 \mu \mathrm{M}$. In this study, we used 0.7 -fold IC50 of quercetin to treat OA chondrocytes (Figure 2A). CCK-8 assays showed that the impaired cell viability induced by IL-1 $\beta$ stimulation was significantly 
attenuated after quercetin treatment (Figure 2B). ELISA results showed that quercetin treatment significantly suppressed the increases in IL-18 and TNF- $\alpha$ levels induced by IL-1 $\beta$ stimulation (Figure $2 \mathrm{C}$ and $\mathrm{D}$ ). Subsequently, both flow cytometry (Figure 2E) and Hoechst staining (Figure $2 \mathrm{~F}$ ) demonstrated that IL-1 $\beta$ stimulation promoted cell apoptosis, and treatment with quercetin could reverse that promoting effect. At the molecular level, the IL-1 $\beta$ induced upregulation of IRAK1, NLRP3, iNOS, COX-2 and Caspase 3 expression was obviously suppressed by quercetin treatment (Figure $2 \mathrm{G}$ and $\mathrm{H}$ ). The decreased levels of NLRP3 expression caused by quercetin treatment in IL-1 $\beta$-treated chondrocytes were further verified by immunofluorescence (Figure $2 \mathrm{G}$ and $\mathrm{H}$ ).

\section{Overexpression of NLRP3 Reversed the Suppressive Effect of Quercetin on IL- I $\beta$ - Induced Rat Chondrocyte Injuries in vitro} Because NLRP3 was downregulated in IL-1 $\beta$-induced rat chondrocytes after quercetin treatment, gain-offunction assays were performed to investigate whether NLRP3 expression was involved in the mechanism by which quercetin helped to protect against IL-1 $\beta$-induced
A

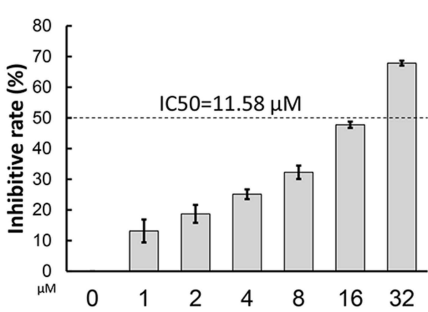

E

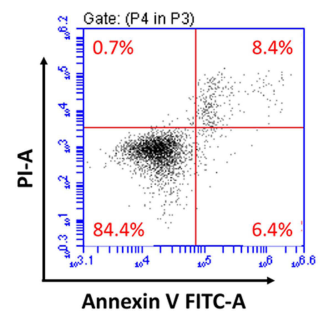

$\mathbf{F}$

Blank

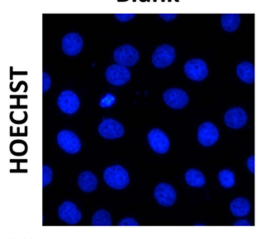

H Blank

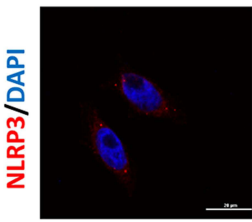

B

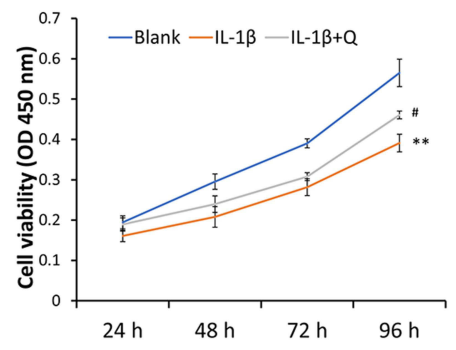

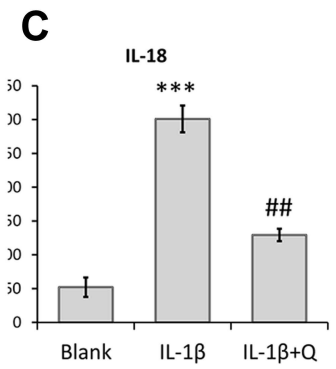

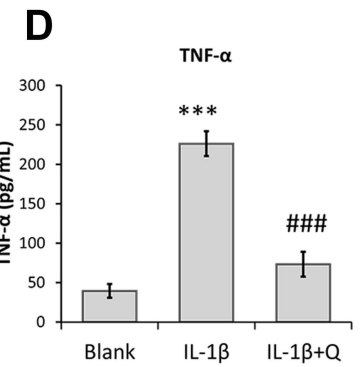

Figure 2 Quercetin attenuated IL-I $\beta$-induced inflammation and the apoptosis of rat chondrocytes. Rat chondrocytes were treated with $8 \mu \mathrm{M}$ quercetin for $2 \mathrm{~h}$, followed by stimulation with IL-I $\beta$. (A) IC50 of OA chondrocytes to quercetin. (B) Chondrocyte viability was analyzed by the CCK-8 assay. (C and D) The levels of pro-inflammatory cytokines (IL-18 and TNF- $\alpha$ ) were determined by ELISA. (E) Cell apoptosis was evaluated by flow cytometry analysis performed with an Annexin V-FITC/propidium iodide (PI) apoptosis kit. (F) Hoechst 33342 staining was performed. Apoptotic cells exhibited morphological changes in the nuclei typical of apoptosis. Images were captured under a fluorescence microscope. (G) The levels of NLRP3, ASC, and IRAKI proteins were examined by Western blotting. (H) NLRP3 immunofluorescence staining. Markedly increased red bright puncta indicate the upregulated expression of NLRP3 (bar: $20 \mu \mathrm{m}$ ). Data represent a mean value \pm standard deviation. ${ }^{\mathrm{p}} \mathrm{p}<0.05$, $* * \mathrm{p}<0.0 \mathrm{I}$, *** $\mathrm{p}<$ 0.001 , compared with blank; ${ }^{\#} \mathrm{p}<0.05,{ }^{\#} \mathrm{p}<0.0 \mathrm{I},{ }^{\# \#} \mathrm{P}<0.00 \mathrm{I}$, compared with IL-I $\beta .{ }^{\$ \$} \mathrm{P}<0.0 \mathrm{I}$, compared with IL-I $\beta+$ quercetin + vector.

Abbreviation: NS, No significance. 
injuries. CCK-8 assays revealed that increased cell viability of IL-1 $\beta$-induced rat chondrocytes that was observed following treatment with quercetin was abolished after NLRP3 overexpression (Figure 3A). Similarly, ELISA, flow cytometry, and Hoechst staining results showed that the decreases in pro-inflammatory cytokine (IL-1 $\beta$ and TNF- $\alpha$ ) levels and apoptosis caused by quercetin treatment in IL-1 $\beta$-induced rat chondrocytes were both reversed by overexpression of NLRP3
(Figure 3B-D, respectively). In addition, quercetin treatment effectively suppressed the production of ROS in IL-1 $\beta$-induced rat chondrocytes, and that affect was also reversed by NLRP3 overexpression (Figure 3E). Western blot analyses further confirmed that NLRP3 overexpression significantly reversed the suppressive effects of quercetin on IRAK1, NLRP3, ASC, iNOS, COX-2 and caspase-3 protein expression in IL-1 $\beta$ induced rat chondrocytes (Figure $3 \mathrm{~F}$ ).
A

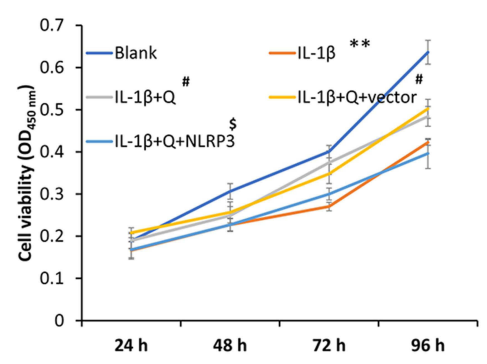

C

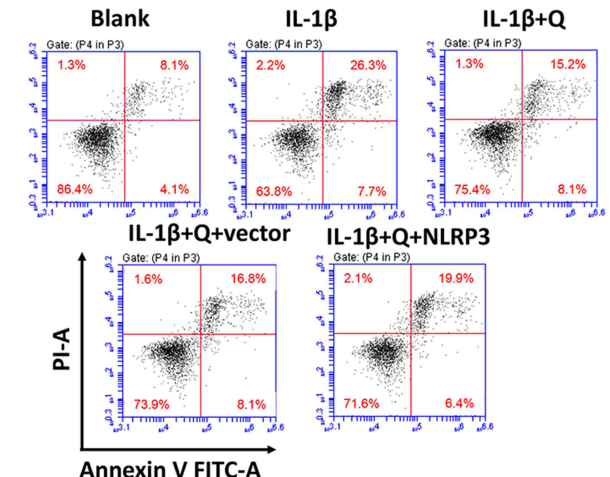

E
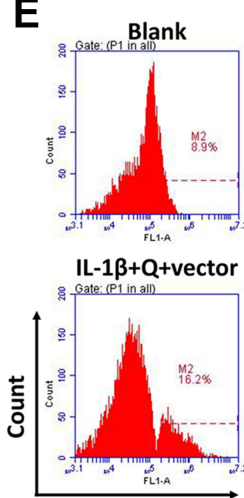

FL1-A
B
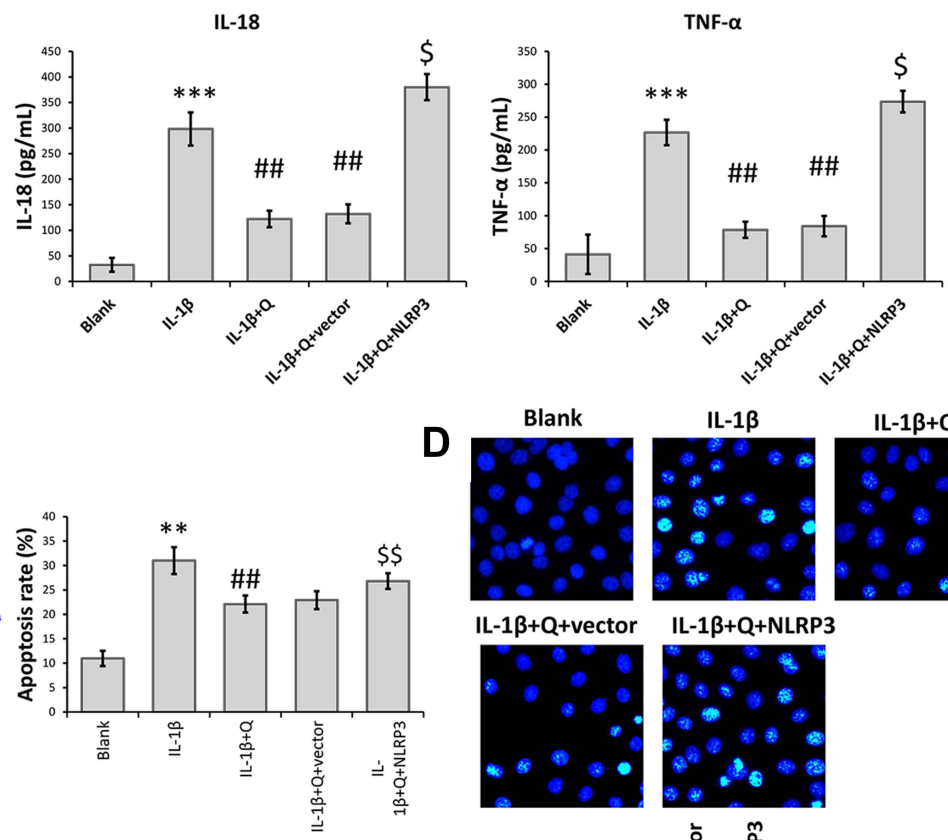

Blank

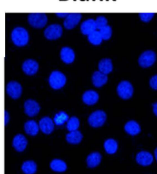

IL-1 $\beta$

IL-1 $\beta+Q$

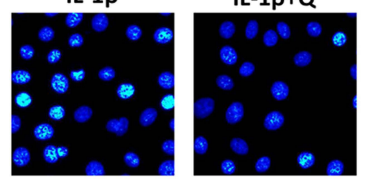

IL-1 $\beta+Q+$ vector

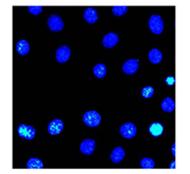

IL-1 $\beta+Q+N L R P 3$

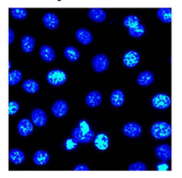

F

ᄒ
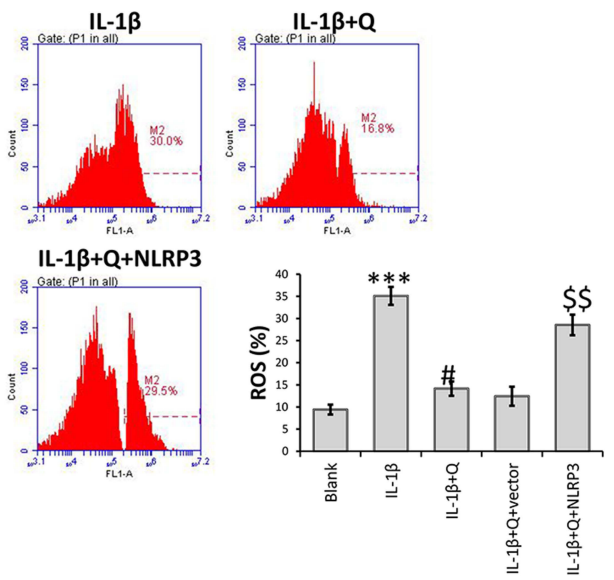

NLRP3

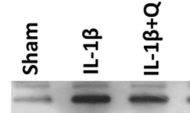

$\begin{array}{lllll}0.23 & 1.24 & 0.79 & 0.66 & 1.33\end{array}$

ASC

$\begin{array}{lllll}1.46 & 1.57 & 0.56 & 0.73 & 1.69\end{array}$

IRAK1
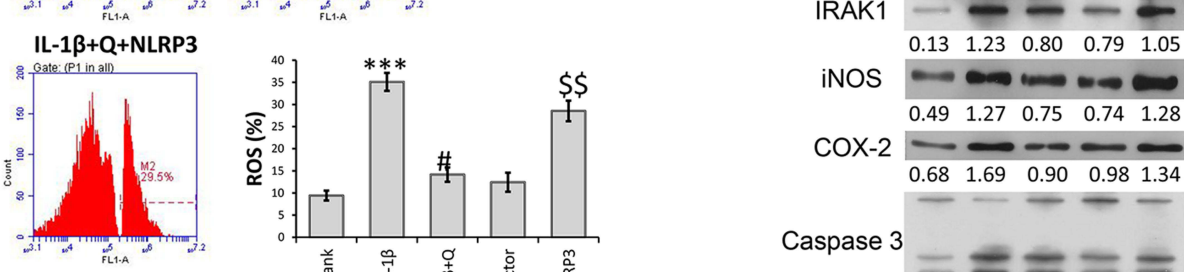

iNOS

$\begin{array}{lllll}0.13 & 1.23 & 0.80 & 0.79 & 1.05\end{array}$

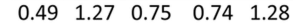

COX-2

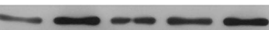

$\begin{array}{lllll}0.68 & 1.69 & 0.90 & 0.98 & 1.34\end{array}$

Caspase 3

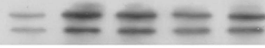

$\begin{array}{lllll}0.33 & 1.37 & 0.93 & 1.19 & 1.16\end{array}$

GAPDH

Figure 3 Overexpression of NLRP3 reversed the suppressive effects of quercetin on IL-I $\beta$-induced rat chondrocyte injuries in vitro. Chondrocytes in the IL-I $\beta+$ quercetin group were transiently transfected with an NLRP3 overexpression plasmid or empty vector. (A) Chondrocyte viability was analyzed by the CCK-8 assay. (B) The levels of pro-inflammatory cytokines (IL-I8 and TNF- $\alpha$ ) were measured by ELISA. (C) Cell apoptosis was evaluated by flow cytometry performed with an Annexin V-FITC/propidium iodide (PI) apoptosis kit. (D) Hoechst 33342 staining was performed. Apoptotic cells exhibited morphological changes in the nuclei typical of apoptosis. Images were captured under a fluorescence microscope. (E) The intracellular levels of ROS in chondrocytes were assessed using a DCFH-DA probe. (F) The levels of NLRP3, ASC, IRAKI, iNOS, COX-2 and caspase 3 proteins were assessed by Western blotting. Data represent a mean value \pm standard deviation. $* * p<0.0$ I, $* * * p<0.00$ I, compared with blank; ${ }^{\#}<0.05,{ }^{\# \#}<0.01$, compared with IL-I $\beta$; ${ }^{\$} \mathrm{P}<0.05,{ }^{\$} \mathrm{P}<0.01$, compared with IL-I $\beta+$ quercetin + vector. 


\section{Quercetin Inhibited IL-I $\beta$-Induced Rat} Chondrocyte Injuries in vitro by

\section{Suppressing the IRAKI/NLRP3 Signaling Pathway}

The above data showed that IRAK1/NLRP3 expression in IL-1 $\beta$-induced rat chondrocytes was decreased after quercetin treatment. We performed rescue experiments to determine whether the protective effect of quercetin in IL-1 $\beta$-stimulated chondrocytes was associated with the IRAK1/NLRP3 signaling pathway. Rat chondrocytes were co-transfected with IRAK1 and si-NLRP3, followed by quercetin treatment and IL- $1 \beta$ stimulation. A series of functional experiments demonstrated that
A

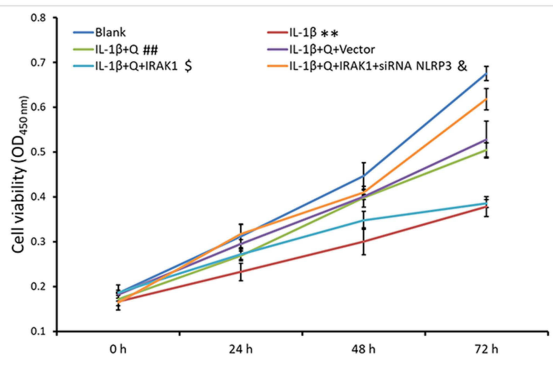

B

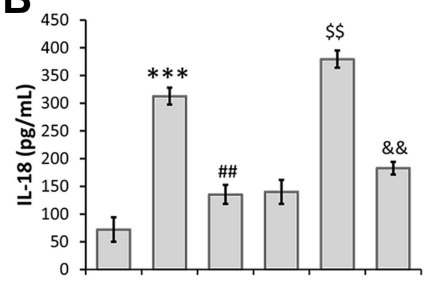

C
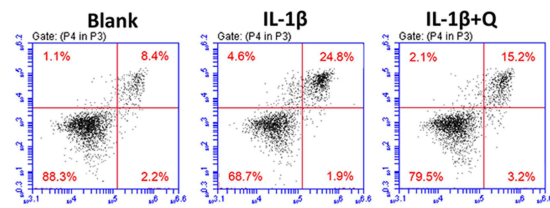

IL-1 $\beta+Q+$ Vector
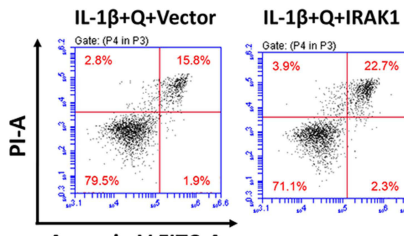

-1 $\beta+Q+I R A K 1$
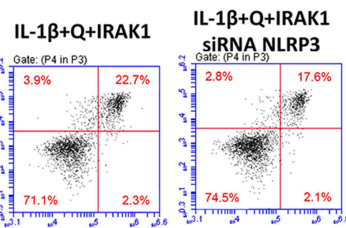

Annexin V FITC-A

E
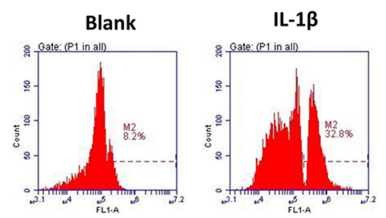

IL- $1 \beta+Q+$ Vector

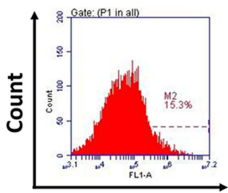

FL1-A
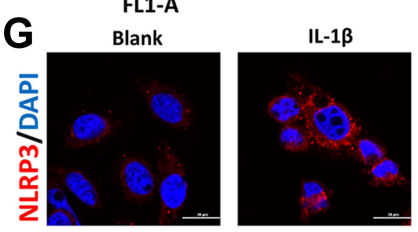
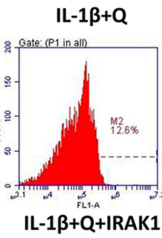
SIRNA NLRP3

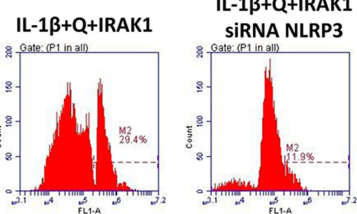

$\mathrm{IL}-1 \beta+\mathrm{Q}$

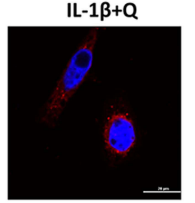

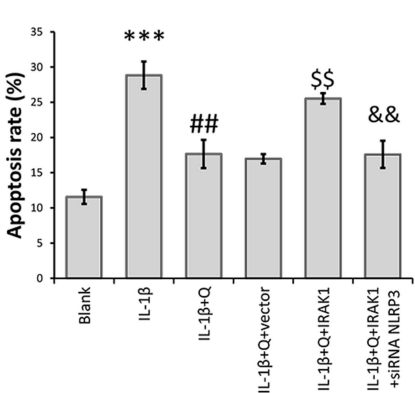

D

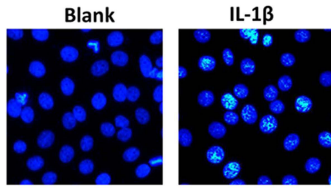

IL-1 $\beta+Q+$ Vector IL-1 $\beta+Q+I R A K 1$
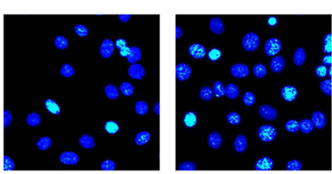

$\mathbf{F}$
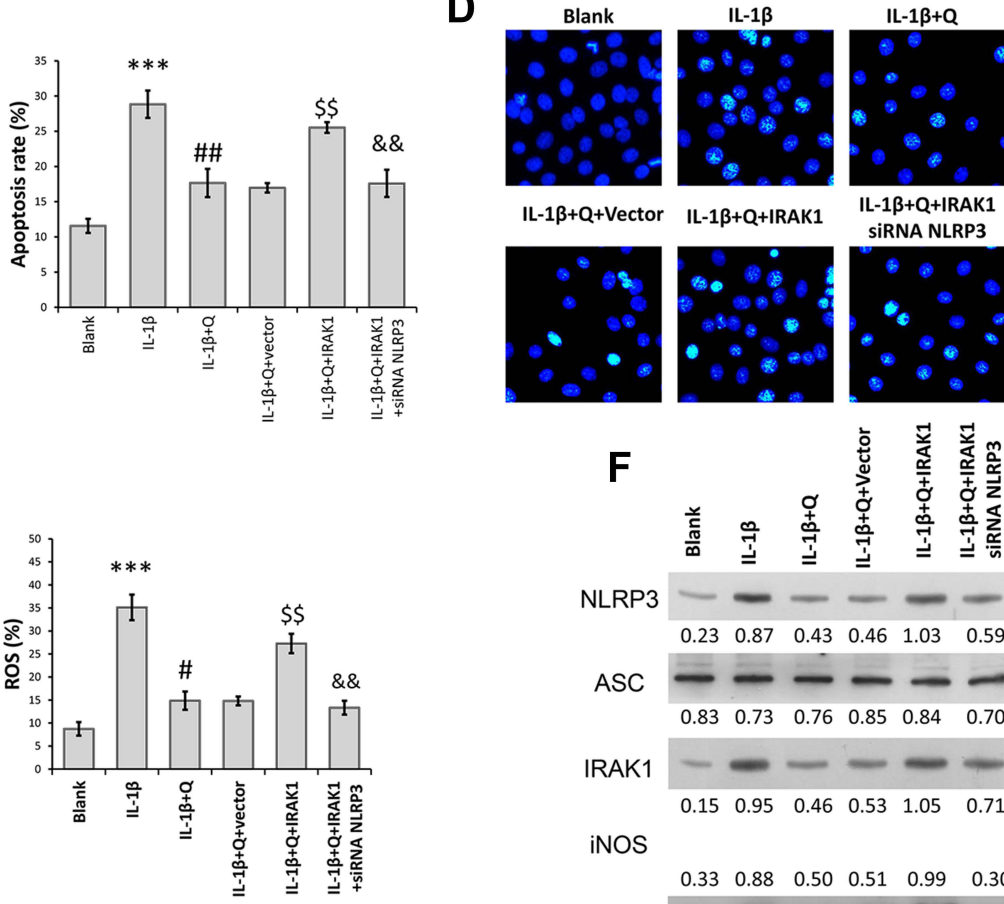

IL-1 $\beta+Q+I R A K 1$

SIRNA NLRP3
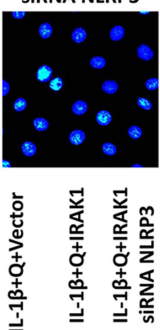

NLRP3

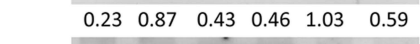

ASC

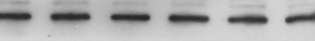

$\begin{array}{llllll}0.83 & 0.73 & 0.76 & 0.85 & 0.84 & 0.70\end{array}$

IRAK1

$0.15 \quad 0.95 \quad 0.46$

iNOS

$\begin{array}{llllll}0.33 & 0.88 & 0.50 & 0.51 & 0.99 & 0.30\end{array}$

COX-2 $=-\infty=$
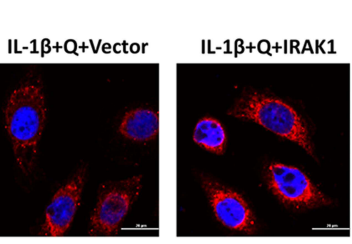

IL- $1 \beta+Q+$ IRAK SIRNA NLRP3

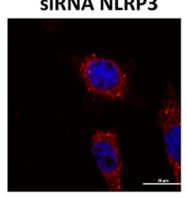

$\begin{array}{llllll}0.42 & 0.99 & 0.68 & 0.81 & 1.06 & 0.41\end{array}$

Caspase 3
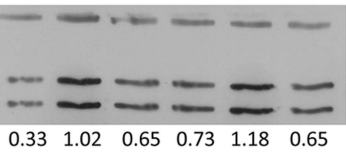

GAPDH

Figure 4 Quercetin inhibited IL-I $\beta$-induced rat chondrocyte injuries in vitro by suppressing the IRAKI/NLRP3 signaling pathway. Chondrocytes in the IL-I $\beta+$ quercetin group were transiently co-transfected with siRNA targeting NLRP3 (si-NLRP3) and an IRAKI overexpression plasmid. (A) Chondrocyte viability was analyzed by the CCK-8 assay. (B) The levels of pro-inflammatory cytokines (IL-18 and TNF- $\alpha$ ) were determined by ELISA. (C) Cell apoptosis was evaluated by flow cytometry performed with an Annexin V-FITC/propidium iodide (PI) apoptosis kit. (D) Hoechst 33342 staining was performed. Apoptotic cells exhibited morphological changes in the nuclei typical of apoptosis. Images were captured under a fluorescence microscope. (E) The intracellular levels of ROS in chondrocytes were assessed using a DCFH-DA probe. (F) The levels of NLRP3, ASC, iNOS, COX-2 and IRAKI proteins were assessed by Western blotting. (G) NLRP3 immunofluorescence staining. Markedly increased red bright puncta indicated the upregulated expression of NLRP3 (bar: $20 \mu \mathrm{m}$ ). Data represent a mean value \pm standard deviation. ${ }^{* *} \mathrm{p}<0.0 \mathrm{I}$, ***p $<0.00 \mathrm{I}$, compared with blank; ${ }^{*} \mathrm{p}<$ $0.05,{ }^{\#} \mathrm{P}<0.01$, compared with IL-I $\beta ;{ }^{\$} \mathrm{P}<0.05,{ }^{\$ \$} \mathrm{P}<0.0 \mathrm{I}$, compared with IL-I $\beta+$ quercetin + vector; ${ }^{\&} \mathrm{P}<0.05$, ${ }^{\& \&} \mathrm{P}<0.0 \mathrm{I}$, compared with IL-I $\beta+$ quercetin + IRAKI. 
overexpression of IRAK1 reversed the suppressive effects of quercetin on IL-1 $\beta$-induced impaired cell viability (Figure 4A), and increased inflammation (Figure 4B), apoptosis (Figure 4C and D), and ROS production (Figure 4E) in injured rat chondrocytes, and those effects were all attenuated by NLRP3 knockdown. Western blot studies further confirmed that the levels of NLRP3 and IRAK1 expression were increased after IRAK1 overexpression, but were decreased by NLRP3 knockdown in the quercetin plus IL-1 $\beta$ treatment group (Figure 4F). The same trends related to NLRP3 expression were detected by immunofluorescence (Figure 4G). These results suggested that quercetin could inhibit inflammation and chondrocyte apoptosis by suppressing IRAK1/NLRP3 signaling in IL-1 $\beta$-treated chondrocytes.

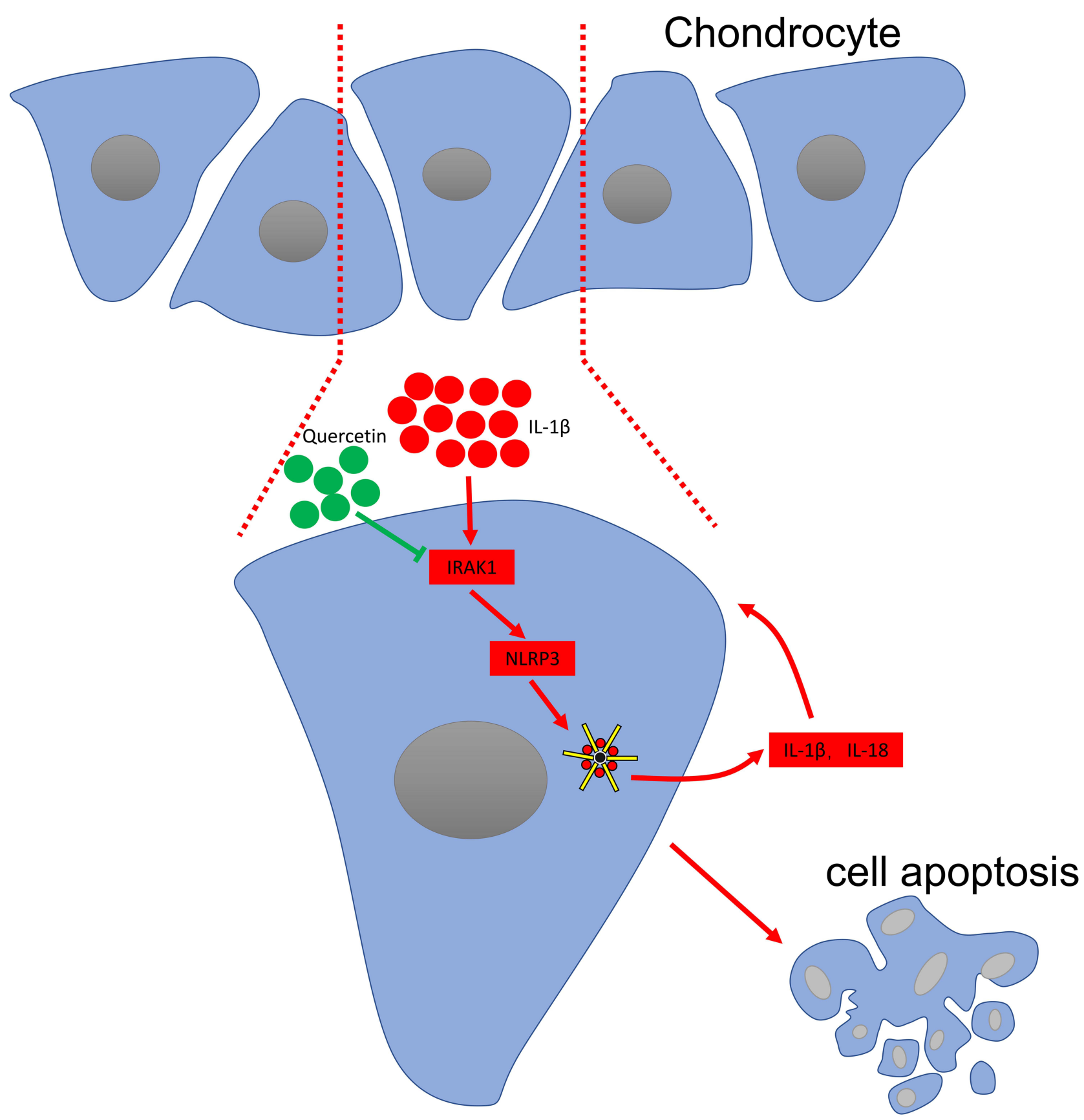

Figure 5 Schematic diagram of the proposed molecular mechanisms underlying the protective effect of quercetin against OA progression. 


\section{Discussion}

Here, our data showed that administration of quercetin attenuated $\mathrm{OA}$ progression in a rat model of $\mathrm{OA}$, as indicated by reduced cartilage destruction, as well as by decreased OARSI scores and inflammation status. Quercetin is a natural product that displays antioxidative properties under conditions of stress in various degenerative diseases. ${ }^{17,18,26}$ Consistent with our in vivo results, Wei et $\mathrm{al}^{27}$ demonstrated that quercetin could improve the degenerative effects of $\mathrm{OA}$ by attenuating oxidative stress responses and inhibiting the degradation of cartilage extracellular matrix. Feng et $\mathrm{al}^{20}$ reported that quercetin could alleviate pathological changes associated with the degeneration of knee joint articular cartilage in OA model rats. In addition, $\mathrm{Hu}$ et $\mathrm{al}^{21}$ reported that intra-articular injection of quercetin alleviated the degradation of cartilage and decreased the apoptosis of chondrocytes in a rat OA model.

Our data further confirmed that quercetin could suppress expression of the IRAK1/NLRP3 pathway and decrease the levels of pro-inflammatory cytokines (IL-1 $\beta$, IL-18, and TNF- $\alpha$ ) in a rat OA model. To confirm the protective effect of quercetin against the progression of OA associated with IRAK1/NLRP3 signaling, we isolated rat chondrocytes and then mimicked $\mathrm{OA}$ chondrocytes in vitro by incubating the isolated chondrocytes with IL$1 \beta$. IL- $1 \beta$ is one of the most prominent mediators of cartilage degradation and joint inflammation, both of which induce a cascade of inflammatory and catabolic events in chondrocytes. ${ }^{28,29}$ We subsequently observed that quercetin attenuated IL- $1 \beta$-induced inflammation and apoptosis in rat chondrocytes. In line with our data, another study showed that theIL-1 $\beta$-induced accumulation of nitric oxide (NO), matrix metalloproteinase (MMP)-3, and MMP-13 could be suppressed by quercetin. ${ }^{19}$ Furthermore, quercetin was shown to inhibit tert-butyl hydroperoxide (TBHP)-induced apoptosis, oxidative stress, and ER stress in chondrocytes. ${ }^{20}$ Quercetin inhibited the expression of inflammatory and catabolic mediators in IL-1 $\beta$-induced rat chondrocytes by suppressing the Akt/NF- $\kappa$ B signaling pathway. ${ }^{21}$

Subsequently, we performed gain-of-function assays to examine whether overexpression of NLRP3 could reverse the suppressive effects of quercetin on IL-1 $\beta$-induced rat chondrocyte injuries in vitro. Those experiments confirmed that IRAK1 overexpression could reverse the suppressive effects of quercetin on IL- $1 \beta$-induced impaired cell viability, increased inflammation, apoptosis, and ROS production, which were all attenuated by NLRP3 knockdown in injured rat chondrocytes. The IRAK1/NLRP3 pathway has been reported to participate in the inflammation and apoptosis associated with OA progression. For example, inhibition of IRAK1 phosphorylation was demonstrated to be involved in the mechanism by which delphinidin inhibits IL- $1 \beta$-induced COX-2 expression and PGE2 production in human chondrocytes. ${ }^{13}$ NLRP3 inflammasomes have been suggested as novel biomarkers for use in managing $\mathrm{OA},{ }^{14}$ because they help to regulate the protective effects of icariin ${ }^{15}$ and curcumin ${ }^{16}$ against OA. Lin et al $^{10}$ showed that primer-dependent activation of the NLRP3 inflammasome occurs independent of direct participation of IRAK-1. Moreover, Kang et al ${ }^{12}$ reported that blockage of TLR4/6-IRAK4/1 signaling to suppress NLRP3 inflammasome activation may protect against fructose-induced cardiac inflammation and fibrosis. Based on this evidence, we speculated that quercetin might help to prevent IL-1 $\beta$-induced rat chondrocyte injuries in vitro by suppressing the IRAK1/NLRP3 signaling pathway.

\section{Conclusion}

In summary, the current study, for the first, showed that quercetin exerts its therapeutic effects on OA by inhibiting the inflammasome involved with IRAK1/NLRP3 inflammatory pathway (Figure 5). However, some limitations of our study are that we did not investigate the possible side effects of quercetin when administered to rats or the mechanism by which the IRAK1/NLRP3 inflammatory pathway affects OA pathogenesis in vivo. However, when taken together, our study suggests quercetin as potential drug for use in limiting the pathological progression of OA.

\section{Disclosure}

The authors report no conflicts of interest in this work.

\section{References}

1. Taruc-Uy RL, Lynch SA. Diagnosis and treatment of osteoarthritis. Prim Care. 2013;40(4):821-36,vii. doi:10.1016/j.pop.2013.08.003

2. Ashford S, Williard J. Osteoarthritis: a review. Nurse Pract. 2014;39 (5):1-8. doi:10.1097/01.NPR.0000445886.71205.c4

3. Eichaker LR, Cho H, Duvall CL, et al. Future nanomedicine for the diagnosis and treatment of osteoarthritis. Nanomedicine. 2014;9 (14):2203-2215. doi: $10.2217 / \mathrm{nnm} .14 .138$

4. Cheng DS, Visco CJ. Pharmaceutical therapy for osteoarthritis. $P M \& R$. 2012;4(5 Suppl):S82-S88. doi:10.1016/j.pmrj.2012.02.009

5. Steinecker-Frohnwieser B, Weigl L, Kullich W, Lohberger B. The disease modifying osteoarthritis drug diacerein is able to antagonize pro inflammatory state of chondrocytes under mild mechanical stimuli. Osteoarthr Cartil. 2014;22(7):1044-1052. doi:10.1016/j. joca.2014.05.008 
6. Qin J, Shang L, Ping AS, et al. TNF/TNFR signal transduction pathway-mediated anti-apoptosis and anti-inflammatory effects of sodium ferulate on IL-1beta-induced rat osteoarthritis chondrocytes in vitro. Arthritis Res Ther. 2012;14(6):R242. doi:10.1186/ar4085

7. Cheng BY, Lau EY, Leung HW, et al. IRAK1 augments cancer stemness and drug resistance via the AP-1/AKR1B10 signaling cascade in hepatocellular carcinoma. Cancer Res. 2018;78 (9):2332-2342. doi:10.1158/0008-5472.CAN-17-2445

8. Wee ZN, Yatim SM, Kohlbauer VK, et al. IRAK1 is a therapeutic target that drives breast cancer metastasis and resistance to paclitaxel. Nat Commun. 2015;6:8746. doi:10.1038/ncomms9746

9. Singer JW, Fleischman A, Al-Fayoumi S, et al. Inhibition of interleukin-1 receptor-associated kinase 1 (IRAK1) as a therapeutic strategy. Oncotarget. 2018;9(70):33416-33439. doi:10.18632/ oncotarget. 26058

10. Lin KM, Hu W, Troutman TD, et al. IRAK-1 bypasses priming and directly links TLRs to rapid NLRP3 inflammasome activation. Proc Natl Acad Sci U S A. 2014;111(2):775-780. doi:10.1073/ pnas. 132029411

11. Fernandes-Alnemri T, Kang S, Anderson C, et al. Cutting edge: TLR signaling licenses IRAK1 for rapid activation of the NLRP3 inflammasome. J Immunol. 2013;191(8):3995-3999. doi:10.4049/ jimmunol.1301681

12. Kang LL, Zhang DM, Ma CH, et al. Cinnamaldehyde and allopurinol reduce fructose-induced cardiac inflammation and fibrosis by attenuating CD36-mediated TLR4/6-IRAK4/1 signaling to suppress NLRP3 inflammasome activation. Sci Rep. 2016;6:27460. doi:10.1038/srep27460

13. Haseeb A, Chen D, Haqqi TM. Delphinidin inhibits IL-1 $\beta$-induced activation of NF- $\kappa \mathrm{B}$ by modulating the phosphorylation of IRAK-1 (Ser376) in human articular chondrocytes. Rheumatology. 2013;52 (6):998-1008. doi:10.1093/rheumatology/kes363

14. McAllister MJ, Chemaly M, Eakin AJ, et al. NLRP3 as a potentially novel biomarker for the management of osteoarthritis. Osteoarthr Cartil. 2018;26(5):612-619. doi:10.1016/j.joca.2018.02.901

15. $\mathrm{Zu} \mathrm{Y,} \mathrm{Mu} \mathrm{Y,} \mathrm{Li} \mathrm{Q,} \mathrm{et} \mathrm{al.} \mathrm{Icariin} \mathrm{alleviates} \mathrm{osteoarthritis} \mathrm{by} \mathrm{inhibiting}$ NLRP3-mediated pyroptosis. J Orthop Surg Res. 2019;14(1):307. doi:10.1186/s13018-019-1307-6

16. Sun Y, Liu W, Zhang H, et al. Curcumin prevents osteoarthritis by inhibiting the activation of inflammasome NLRP3. J Interferon Cytokine Res. 2017;37(10):449-455. doi:10.1089/jir.2017.0069

17. de Oliveira MR, Nabavi SM, Braidy N, et al. Quercetin and the mitochondria: a mechanistic view. Biotechnol Adv. 2016;34 (5):532-549. doi:10.1016/j.biotechadv.2015.12.014

18. Kanter M, Aktas C, Erboga M. Protective effects of quercetin against apoptosis and oxidative stress in streptozotocin-induced diabetic rat testis. Food Chem Toxicol. 2012;50(3-4):719-725. doi:10.1016/j. fct.2011.11.051
19. Qiu L, Luo Y, Chen X. Quercetin attenuates mitochondrial dysfunction and biogenesis via upregulated AMPK/SIRT1 signaling pathway in OA rats. Biomed Pharmacother. 2018;103:1585-1591. doi:10.1016/j.biopha.2018.05.003

20. Feng K, Chen Z, Pengcheng L, et al. Quercetin attenuates oxidative stress-induced apoptosis via SIRT1/AMPK-mediated inhibition of ER stress in rat chondrocytes and prevents the progression of osteoarthritis in a rat model. $J$ Cell Physiol. 2019;234(10):18192-18205. doi:10.1002/jcp.28452

21. Hu Y, Gui Z, Zhou Y, et al. Quercetin alleviates rat osteoarthritis by inhibiting inflammation and apoptosis of chondrocytes, modulating synovial macrophages polarization to M2 macrophages. Free Radic Biol Med. 2019;145:146-160. doi:10.1016/j. freeradbiomed.2019.09.024

22. Stoop R, Buma P, van der Kraan PM, et al. Type II collagen degradation in articular cartilage fibrillation after anterior cruciate ligament transection in rats. Osteoarthr Cartil. 2001;9(4):308-315. doi:10.1053/joca.2000.0390

23. Tang Q, Zheng G, Feng Z, et al. Trehalose ameliorates oxidative stress-mediated mitochondrial dysfunction and ER stress via selective autophagy stimulation and autophagic flux restoration in osteoarthritis development. Cell Death Dis. 2017;8(10):e3081. doi:10.1038/ cddis. 2017.453

24. Pritzker KP, Gay S, Jimenez SA, et al. Osteoarthritis cartilage histopathology: grading and staging. Osteoarthr Cartil. 2006;14(1):13-29. doi:10.1016/j.joca.2005.07.014

25. Wang SN, Xie GP, Qin CH, et al. Aucubin prevents interleukin-1 beta induced inflammation and cartilage matrix degradation via inhibition of NF-kappaB signaling pathway in rat articular chondrocytes. Int Immunopharmacol. 2015;24(2):408-415. doi:10.1016/j. intimp.2014.12.029

26. Roslan J, Giribabu N, Karim K, Salleh N. Quercetin ameliorates oxidative stress, inflammation and apoptosis in the heart of streptozotocin-nicotinamide-induced adult male diabetic rats. Biomed Pharmacother. 2017;86:570-582. doi:10.1016/j. biopha.2016.12.044

27. Wei B, Zhang Y, Tang L, et al. Protective effects of quercetin against inflammation and oxidative stress in a rabbit model of knee osteoarthritis. Drug Dev Res. 2019;80(3):360-367. doi:10.1002/ ddr. 21510

28. Liu Y, Zhu H, Yan X, et al. Endoplasmic reticulum stress participates in the progress of senescence and apoptosis of osteoarthritis chondrocytes. Biochem Biophys Res Commun. 2017;491 (2):368-373. doi:10.1016/j.bbrc.2017.07.094

29. Zwerina J, Redlich K, Polzer K, et al. TNF-induced structural joint damage is mediated by IL-1. Proc Natl Acad Sci U S A. 2007;104 (28):11742-11747. doi:10.1073/pnas.0610812104
Journal of Inflammation Research

\section{Publish your work in this journal}

The Journal of Inflammation Research is an international, peerreviewed open-access journal that welcomes laboratory and clinica findings on the molecular basis, cell biology and pharmacology of inflammation including original research, reviews, symposium reports, hypothesis formation and commentaries on: acute/chronic inflammation; mediators of inflammation; cellular processes; molecular mechanisms; pharmacology and novel anti-inflammatory drugs; clinical conditions involving inflammation. The manuscript management system is completely online and includes a very quick and fair peerreview system. Visit http://www.dovepress.com/testimonials.php to read real quotes from published authors. 